
\title{
$\begin{array}{ll}\text { Research Square } & \text { Preprints are preliminary reports that have not undergone peer review. } \\ \text { They should not be considered conclusive, used to inform clinical practice, } & \text { or referenced by the media as validated information. }\end{array}$
}

\section{Infections caused by the Delta variant (B.1.617.2) of SARS-CoV-2 are associated with increased viral loads compared to infections with the Alpha variant (B.1.1.7) or non-Variants of Concern.}

Christian von Wintersdorff

Maastricht UMC+

Jozef Dingemans ( $\sim$ jozef.dingemans@mumc.nl)

Maastricht UMC+ https://orcid.org/0000-0001-8079-3087

Lieke van Alphen

Department of Medical Microbiology, Care and Public Health Research Institute (CAPHRI), Maastricht University Medical Center+, Maastricht, The Netherlands.

\section{Petra Wolffs}

Maastricht University Medical Center

Brian van der Veer

Maastricht UMC+

Christian Hoebe

South Limburg Public Health Service

Paul Savelkoul

Maastricht University Medical Centre

\section{Article}

Keywords: SARS-CoV-2, Delta variant, Viral load, Variant of Concern

Posted Date: August 4th, 2021

DOl: https://doi.org/10.21203/rs.3.rs-777577/v1

License: (c) (1) This work is licensed under a Creative Commons Attribution 4.0 International License. Read Full License 


\section{Abstract}

There has been a growing body of evidence that the severe acute respiratory syndrome coronavirus 2 (SARS-CoV-2) Delta variant (B.1.617.2) shows enhanced transmissibility and increased viral loads compared to other variants. A recent study has even suggested that respiratory samples from people infected with the Delta variant can harbor up to 1000 times higher viral loads compared to samples with variants that are more closely related to the original Wuhan strain, although the sample size of this study was very limited. Here, we have compared the viral load in 16,185 samples that were obtained during which non-VOC, the Alpha (B.1.1.7) or Delta variant (B.1.617.2) were dominant as evidenced by genomic surveillance. We found that the Delta variant contained about 4 -fold higher viral loads compared to the non-VOC or Alpha variants.

\section{Introduction}

During the ongoing COVID-19 pandemic, various variants of SARS-CoV-2 have emerged. Some of these variants have been labelled as variants of interest (VOI) or variants of concern (VOC) by the WHO due to their potentially increased transmissibility, disease severity or immune escape characteristics ${ }^{1}$. The B.1.1.7 or Alpha variant was shown to have a greatly increased transmissibility ${ }^{2}$ and rapidly became the dominant circulating SARS-CoV-2 variant in many countries including The Netherlands. Recently, there has been much concern about the B.1.617.2 or Delta variant, which appears to be even more highly transmissible than the Alpha variant ${ }^{3,4,5}$ and is also linked to possible immune escape ${ }^{6,7,8}$. In many countries, the Delta variant has either already replaced the Alpha variant as the dominant variant or is rapidly gaining ground and has causes major resurgences in reported cases.

As the Delta variant established itself as the new dominant SARS-CoV-2 variant in The Netherlands, a seemingly increase in detected viral loads was noticed during routine diagnostic testing at our department. Increased viral loads in infected persons could potentially contribute to the increased transmissibility of the Delta variant. In this study, we investigated the link between viral loads of the Delta and other SARS-CoV-2 variant in infected persons.

\section{Results}

\section{Viral load comparison based on variant-dominant time periods}

In the first comparison, three distinct time periods were determined in which certain SARS-CoV-2 variants were dominant in our testing population in South-Limburg, the Netherlands. These periods were labelled as the non-VOC period (December 2020 - February 2021), Alpha period (March 2021 - June 2021) and Delta period (July 2021). The prevalence of SARS-CoV-2 variants in these time periods was based on next-generation sequencing data of our genomic surveillance program (Figure 1), which is part of the Dutch national SARS-CoV-2 surveillance program. 
Figure 2A-B shows that the viral load was significantly higher in the time period in which infections were predominantly caused by the Delta variant (median CT 18 or $6.55 \log _{10} \mathrm{c} / \mathrm{mL}$, Table 1 ), when compared to the non-VOC or Alpha periods (median CT 20 or $5.98 \log _{10} \mathrm{c} / \mathrm{mL}$ ).

Table 1: comparison of CT values and viral loads between non-VOC, Alpha and Delta variants of SARS-CoV-2.

\begin{tabular}{|l|l|l|l|l|l|l|l|l|}
\hline & & \multicolumn{5}{|c|}{ CT value } & \multicolumn{3}{c|}{ Viral Load } \\
\hline & $\mathrm{N}$ & Median & Mean & 95\% CI & Median & Mean & 95\% CI \\
\hline \multicolumn{7}{|c|}{ Time periods } \\
\hline Non-VOC period & 8630 & 20 & 22.31 & $22.17-22.45$ & 5.98 & 5.38 & $5.34-5.42$ \\
\hline Alpha period & 7177 & 20 & 22.26 & $22.10-22.43$ & 5.98 & 5.41 & $5.36-5.45$ \\
\hline Delta period & 378 & 18 & 20.28 & $19.58-20.98$ & 6.55 & 5.98 & $5.80-6.16$ \\
\hline \multicolumn{7}{|l|}{ WGS confirmed variants } \\
\hline Non-VOC & 631 & 19 & 19.48 & $19.14-19.81$ & 6.26 & 6.13 & $6.03-6.22$ \\
\hline Alpha & 1240 & 18 & 18.81 & $18.58-19.04$ & 6.55 & 6.32 & $6.25-6.38$ \\
\hline Delta & 87 & 16 & 16.71 & $15.88-17.54$ & 7.11 & 6.91 & $6.68-7.14$ \\
\hline
\end{tabular}

\section{Viral load comparison of sequence-confirmed variants}

Since the tested SARS-CoV-2 variants in the defined time periods for non-VOC, Alpha and Delta are based on assumption and will also include a small portion of different variants, we aimed to confirm these findings by comparing viral loads of only sequence-confirmed variants. To this end, viral loads of a subset of WGS-confirmed non-VOC $(n=631)$, Alpha $(n=1240)$ and Delta $(n=87)$ variants were compared (table 1, figure 2C-D). These results confirmed that there was an overall higher viral load in the Delta variant group (median CT 16 or $7.11 \log _{10} \mathrm{c} / \mathrm{mL}$, Table 1) when compared to the non-VOC (median CT 19 or $6.26 \log _{10} \mathrm{c} / \mathrm{mL}$, Table 1) or Alpha variant (median CT 18 or $6.55 \log _{10} \mathrm{c} / \mathrm{mL}$, Table 1). Additionally, the viral load of the Alpha variant group was increased compared to the non-VOC group.

\section{Discussion}

Recent studies have indicated that the delta variant is associated with increased viral loads, implying enhanced infectivity of this variant $3,4,5$. Nevertheless, the limited size of datasets used in these studies as well as the different diagnostic methods could lead to bias and preliminary conclusions with regards to the viral load and infectivity that is associated with the delta variant. In this study we have compared the CT-values and viral load obtained from samples that were tested positive during the time periods during which the non-VOC, alpha and delta variants were dominant, as evidenced by genomic surveillance of SARS-CoV-2 in the South Limburg region of The Netherlands. It was found that samples 
that were tested positive since the delta variant became dominant contained significantly higher loads of SARS-CoV-2 compared to samples that were tested during which the non-VOC or alpha variant were dominant. Based on the standard curve used for conversion of CT values to viral loads (Figure S1A), it is estimated that samples harboring the delta variant contain about 4-fold higher loads of SARS-CoV-2 compared to samples harboring non-VOC or the alpha variant. These observations were confirmed using a subset of WGS-confirmed samples in which samples containing the delta variant harbored significantly higher loads of SARS-CoV-2 compared to their non-VOC (about 7-fold) or alpha variant (about 4-fold) counterparts. It has to be noted that the delta variant became dominant at the moment that $>60 \%$ of the population received at least one dose and $>35 \%$ of the total population was fully vaccinated in The Netherlands, leaving open the possibility that the observed differences in viral load might even be more pronounced in non-vaccinated individuals. Interestingly, when using WGS-confirmed data, which consists of samples < CT30, a significantly higher load was observed for samples harboring the alpha variant compared to the non-VOC variant. When performing the same analysis by only including samples $<$ CT30 for non-WGS confirmed samples, there was also a moderate increased viral load observed for samples obtained during the period during which the alpha variant was dominant versus samples collected during the non-VOC period. Recently, it was reported that samples from people who are infected with the delta variant harbor up to 1000 times higher viral loads than people infected with the clade $19 \mathrm{~A} / 19 \mathrm{~B}$ viruses ${ }^{4}$. We did not observe such differences compared to the non-VOC or alpha variant, neither in CT value, nor in estimated viral load. Nevertheless, it has to be noted that the former study ${ }^{4}$ comprised a much smaller dataset (62 samples harboring delta variant vs 63 samples harboring clade 19A/19B isolates) which was part of an outbreak, used a different RNA isolation method and PCR assay and compared the delta variant to a mixture of samples harboring nextstrain clade 19A/19B variants, which did not harbor the D614G mutation that enhances infectivity $9,10,11$ and were much closer related to the original Wuhan strain than the non-VOC isolates in our study. One of the strengths of this study is the large dataset of samples of which the great majority was tested using the same or a highly comparable workflow (Figure S1). Furthermore, follow-up samples obtained from the same subject were filtered out of the dataset as much as possible, to avoid bias. Finally, extensive genomic surveillance in the region allowed us to accurately estimate the dominant period for each variant as WGS of a subset of samples confirmed the observed trends. Limitations of this study can be found in the fact that the available dataset of the delta variant is still limited in size compared to the other datasets and the fact that the non-VOC group consists of many different pangolin lineages.

In conclusion, our study shows that samples from individuals that are infected with the delta variant harbor about 4-fold higher loads of SARS-CoV-2 compared to individuals that are infected with non-VOC or the Alpha variant, which is significantly lower than previously reported ${ }^{4}$.

\section{Methods}

\section{Clinical samples}


In this study, nasopharyngeal samples that were tested positive for SARS-CoV-2 were included. Samples ( $n=16,185$ ) were collected from December 2020 until July 2021 by the South Limburg Public Health Service $(n=13,927)$, nursing homes $(n=1,932)$, general practitioners $(n=226)$ and commercial parties $(n$ $=100$ ). Samples from patients and personnel were excluded as they were regularly tested and could introduce bias in the dataset. All nasopharyngeal swabs were immediately deposited in $3 \mathrm{~mL}$ GLY viral transport medium (Mediaproducts BV) after collection and stored under the same conditions prior to testing.

\section{RNA extraction \& RT-qPCR}

RNA extraction was performed mainly by using the chemagic Viral DNA/RNA 300 Kit H96 kit (Perkin Elmer) according to the manufacturer's instructions and was eluted in $100 \mu \mathrm{L}$. A small fraction (6\%) of extractions were performed by using the MagNA Pure 96 DNA and Viral NA Small Volume Kit (Roche Diagnostics) according to the manufacturer's instructions and was eluted in $100 \mu \mathrm{L}$. Both extraction systems were found to yield highly comparable RNA concentrations, leading to comparable viral loads and limits of detection.

All RT-qPCR data was produced at the department of Medical Microbiology, Maastricht University Medical Center + on Quantstudio 5 systems (Thermofisher). Amplification was performed by multiplex PCR, using the $\mathrm{E}$ gene target ${ }^{12}$ in addition to the $\mathrm{N} 1$ target $^{13}$ and $\mathrm{mCMV}$-ie as internal control. Concentrations for the multiplex PCR were $400 \mathrm{nM}$ primer and $200 \mathrm{nM}$ probe for both the $\mathrm{E}$ and $\mathrm{N} 1$ targets and $300 \mathrm{nM}$ primer (sense: CAACATTGACCACGCACTAGATG; antisense: TTAAACTCCCCAGGCAATGAA) and $200 \mathrm{nM}$ probe (TCTTGGCCCATGCGGCACG ) for the internal control. The assay consisted of $5 \mu$ l of TaqPath 1-Step RTqPCR Master Mix (Thermofisher), $10 \mu \mathrm{l}$ RNA eluate and $5 \mu$ of primer/probe mix. Cycling conditions were 2 min at $25^{\circ} \mathrm{C}, 15 \mathrm{~min}$ at $50^{\circ} \mathrm{C}, 2 \mathrm{~min}$ at $95^{\circ} \mathrm{C}$ followed by 42 cycles of $3 \mathrm{~s}$ at $95^{\circ} \mathrm{C}$ and $30 \mathrm{~s}$ at $60^{\circ} \mathrm{C}$. The lowest obtained CT value of both targets was reported (which was the $\mathrm{N} 1$ gene in the great majority of tests).

\section{Next-generation sequencing of SARS-CoV-2-positive samples}

Samples were stored at -80 degrees Celsius until RNA was isolated for sequencing. For RNA extraction, 90 $\mu \mathrm{l}$ of sample was mixed with $90 \mu \mathrm{l}$ of Chemagic Viral Lysis Buffer (Perkin-Elmer), followed by extraction using the MagNA Pure 96 DNA and Viral NA Small Volume Kit 96 (Roche, Germany) on the MagNA Pure 96 system (Roche, Germany), without the addition of an internal extraction control.

Sequencing was performed using the PCR tiling of SARS-CoV-2 virus with Native Barcoding Expansion 96 (EXP-NBD196) protocol (Version: PTCN_9103_v109_revH_13Jul2020) of Oxford Nanopore technologies, with minor modifications and using the primers previously published by Oude Munnink et al. ${ }^{14}$ Briefly, the only modifications were extending the barcode and adaptor ligation steps up to $60 \mathrm{~min}$ and loading 48 samples per flow cell. 
Bioinformatic analysis was performed using an in-house developed pipeline MACOVID that is based on Artic v1.1.3. In brief, short and obvious chimeric reads are filtered with Cutadapt v2.5. The filtered reads were mapped to the reference genome MN908947.3 with Minimap2 v2.17 and quality checked with "align_trim" function of Artic v1.1.3. Mapped reads were split per primer pool using Samtools v1.9 and a consensus was created per primer pool with Medaka v1.0.3. Variants were called using Medaka v1.0.3 and Longshot v0.4.1. Low coverage regions ( $<30 \mathrm{x})$ were masked with "artic_make_depth_mask" function of Artic v1.1.3. A preconsensus was made with "artic_mask" and the final consensus sequence was made with bcftools v1.10.2. Documentation and source code are available from https://github.com/MUMCMEDMIC/MACOVID under MIT license. The consensus sequences were used to construct a phylogenetic tree with the ncov pipeline $v 5$ of nextstrain. Pangolin lineages were assigned using the Pangolin COVID19 Lineage Assigner web application on https://pangolin.cog-uk.io/.

\section{Statistics}

The analysis of data was performed using Graphpad Prism version 5.04 via the Kruskal-Wallis and posthoc Dunn's Multiple Comparison tests. $\mathrm{P}<0.05$ was considered to be statistically significant.

Power analysis was performed using R statistical software, version 3.6.2 (R Foundation for Statistical Computing, Vienna, Austria). The a priori calculated sample size to detect small differences (Cohen's $f$ value 0.1 ) was 322 samples per group to reach a power of 0.8 , whereas the calculated sample size to detect medium differences (Cohen's $\mathrm{f}$ value 0.25 ) was 52 samples per group to reach a power of 0.8 .

\section{Declarations}

\section{Ethical Statement}

The Medical Review Ethics Committee of the Maastricht UMC+ confirmed that the Medical Research Involving Human Subjects Act (WMO) does not apply to the above mentioned study and that an official approval of this study by the committee is not required (METC reference number 2021-2838).

\section{Competing interests}

None to report.

\section{Authors' contributions}

Christian J.H. von Wintersdorff and Jozef Dingemans analyzed data and equally contributed to the writing of the manuscript. Lieke B. van Alphen, Petra F.G. Wolffs contributed to the writing of the manuscript. Brian M.J.W. van der Veer performed bioinformatics analysis of sequencing data and contributed to the writing of the manuscript. Christian J.P.A. Hoebe and Paul H.M. Savelkoul contributed to the writing of the manuscript.

\section{References}


1. Harvey WT, et al. SARS-CoV-2 variants, spike mutations and immune escape. Nat Rev Microbiol, (2021).

2. Davies NG, et al. Estimated transmissibility and impact of SARS-CoV-2 lineage B.1.1.7 in England. Science 372, (2021).

3. Campbell F, et al. Increased transmissibility and global spread of SARS-CoV-2 variants of concern as at June 2021. Euro Surveill 26, (2021).

4. Li B, et al. Viral infection and transmission in a large, well-traced outbreak caused by the SARS-CoV-2 Delta variant. medRxiv, 2021.2007.2007.21260122 (2021).

5. Brown CM, et al. Outbreak of SARS-CoV-2 Infections, Including COVID-19 Vaccine Breakthrough Infections, Associated with Large Public Gatherings - Barnstable County, Massachusetts, July 2021. MMWR Morb Mortal Wkly Rep, (2021).

6. Lopez Bernal J, et al. Effectiveness of Covid-19 Vaccines against the B.1.617.2 (Delta) Variant. $N$ Engl J Med, (2021).

7. Planas D, et al. Reduced sensitivity of infectious SARS-CoV-2 variant B.1.617.2 to monoclonal antibodies and sera from convalescent and vaccinated individuals. bioRxiv, 2021.2005.2026.445838 (2021).

8. Wall EC, et al. Neutralising antibody activity against SARS-CoV-2 VOCs B.1.617.2 and B.1.351 by BNT162b2 vaccination. The Lancet, (2021).

9. Korber B, et al. Tracking Changes in SARS-CoV-2 Spike: Evidence that D614G Increases Infectivity of the COVID-19 Virus. Cel/ 182, 812-827 e819 (2020).

10. Plante JA, et al. Spike mutation D614G alters SARS-CoV-2 fitness. Nature 592, 116-121 (2021).

11. Zhang L, et al. SARS-CoV-2 spike-protein D614G mutation increases virion spike density and infectivity. Nat Commun 11, 6013 (2020).

12. Corman VM, et al. Detection of 2019 novel coronavirus (2019-nCoV) by real-time RT-PCR. Euro Surveill 25, (2020).

13. Lu X, et al. US CDC Real-Time Reverse Transcription PCR Panel for Detection of Severe Acute Respiratory Syndrome Coronavirus 2. Emerg Infect Dis 26, 1654-1665 (2020).

14. Oude Munnink BB, et al. Rapid SARS-CoV-2 whole-genome sequencing and analysis for informed public health decision-making in the Netherlands. Nat Med 26, 1405-1410 (2020).

\section{Figures}




\section{Regional surveillance}

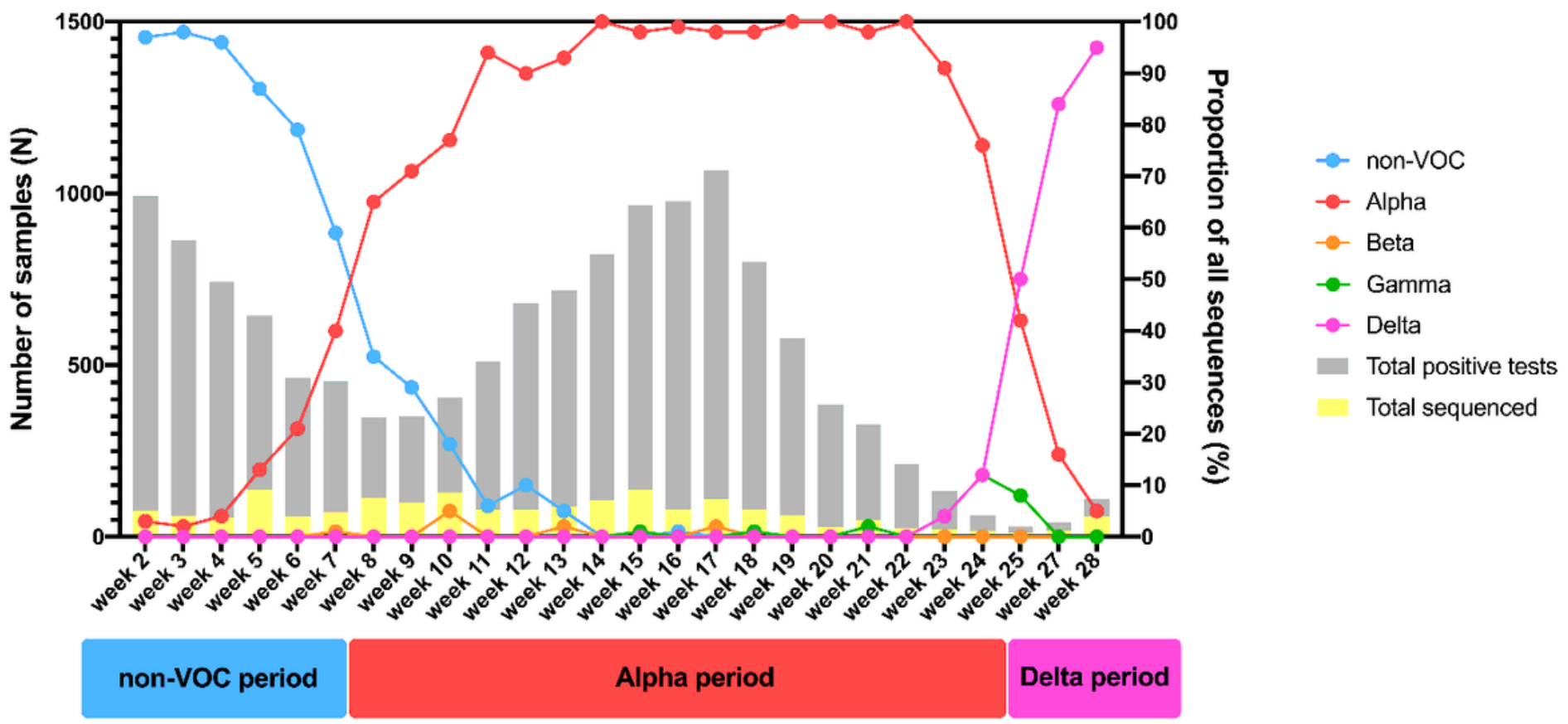

Figure 1

Distribution of circulating variants in the South Limburg region in function of time. The period during which a particular variant was dominant ( $>50 \%$ of sequenced samples) is indicated below. Lines represent the relative proportion of each variant, whereas bars represent the total number of samples that were tested positive (grey) or were sequenced (yellow) in function of time. 


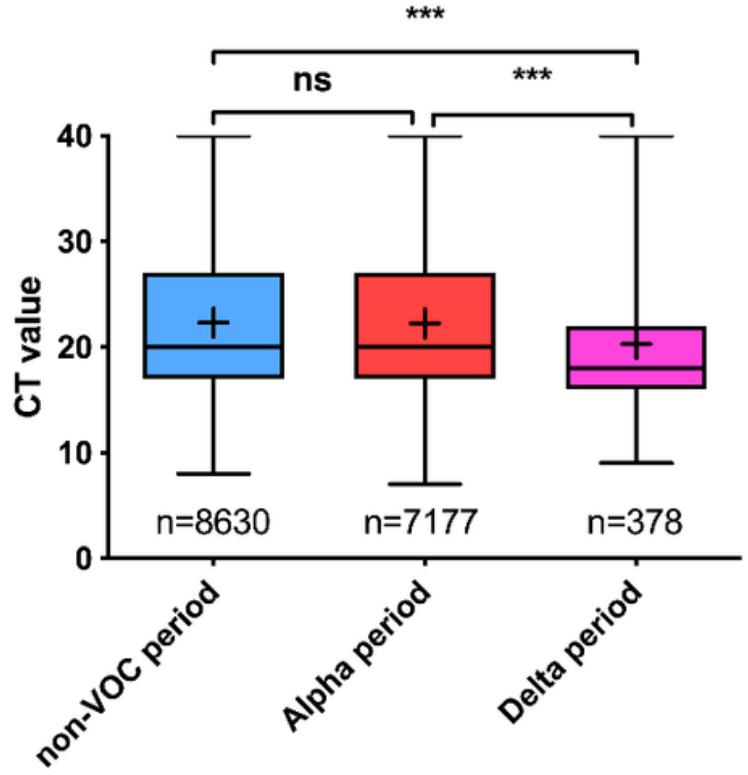

Dominant variant time periods
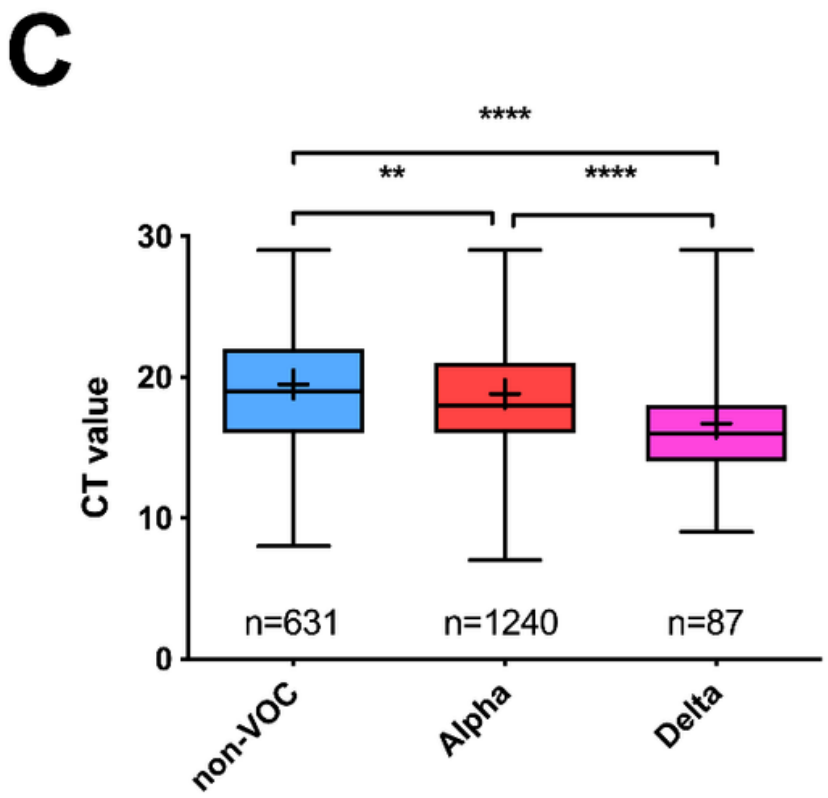

WGS-confirmed

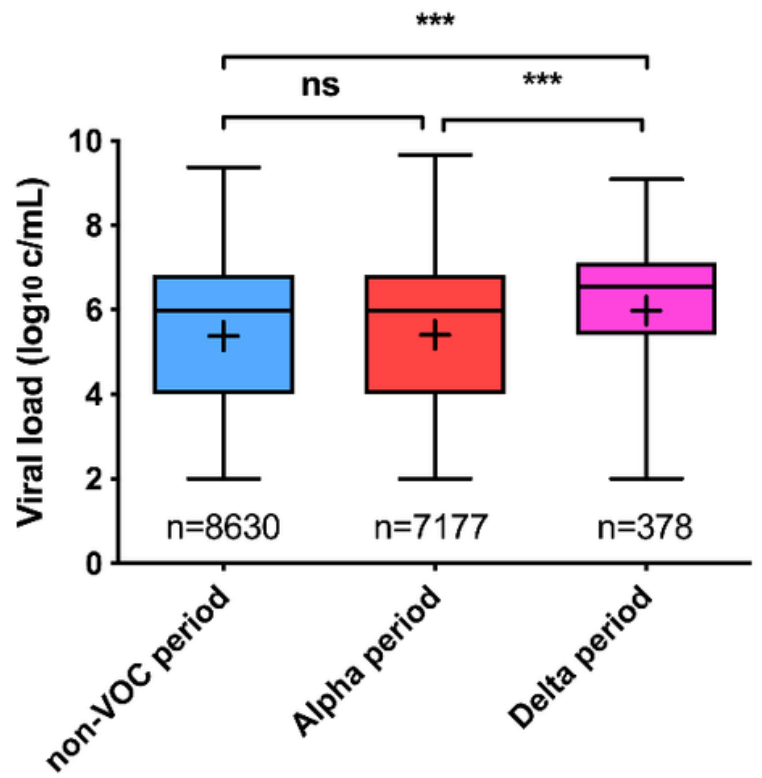

Dominant variant time periods
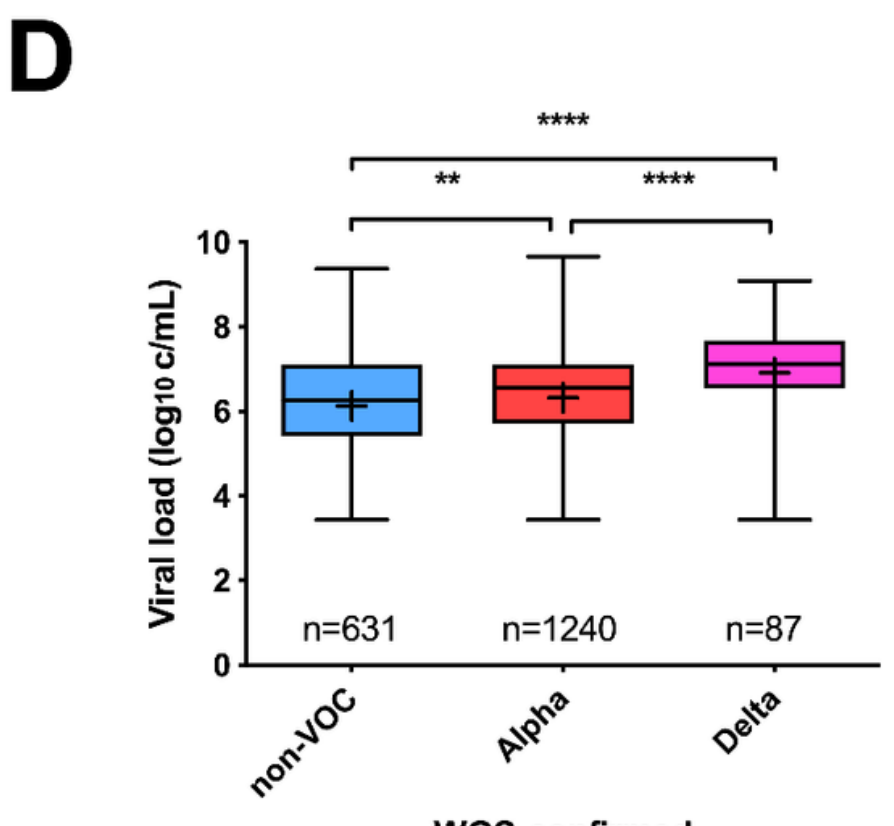

WGS-confirmed

Figure 2

CT values (A) and viral loads (B) per time period dominated by non-VOC (Dec 2020 - Feb 2020), Alpha (Mar 2020 - Jun 2020) and Delta (Jul 2020) SARS-CoV-2 variants. CT values (C) and viral loads (D) per WGS confirmed SARS-CoV-2 variants. Ns: not significant, $* *$ : $p<0.01$, $* \star *: P<0.001, * \star \star *: p<0.0001$. All grouped analyses had a power $>0.99$.

\section{Supplementary Files}


This is a list of supplementary files associated with this preprint. Click to download.

- Supplementarymaterial.pdf 